\title{
Tackling ionospheric scintillation threat to GNSS in Latin America
}

\author{
Vadakke Veettil Sreeja ${ }^{1, *}$, Marcio Aquino ${ }^{1}$, Biagio Forte ${ }^{1,6}$, Zeynep Elmas ${ }^{1}$, Craig Hancock ${ }^{1}$, \\ Giorgiana De Franceschi ${ }^{2}$, Lucilla Alfonsi ${ }^{2}$, Luca Spogli ${ }^{2}$, Vincenzo Romano ${ }^{2}$, Bruno Bougard ${ }^{3}$, \\ Joao Francisco Galera Monico ${ }^{4}$, Andrzej W. Wernik ${ }^{5}$, Jean-Marie Sleewaegen ${ }^{3}$, Andrea Cantó ${ }^{7}$, \\ and Elcia Ferreira Da Silva ${ }^{8}$ \\ 1 Institute of Engineering Surveying and Space Geodesy, University of Nottingham, Nottingham, NG7 2RD, UK \\ *corresponding author: e-mail: v.sreeja@gmail.com \\ 2 Istituto Nazionale di Geofisica e Vulcanologia, Vigna Murata 605, 00143 Rome, Italy \\ 3 Septentrio N. V., Greenhill Campus, Interleuvenlaan 15G, 3001 Leuven, Belgium \\ 4 Faculdade de Ciências e Tecnologia, Departamento de Cartografia, Universidade Estadual Paulista Julio de Mesquita Filho, \\ Rua Roberto Simonsen, 305, Presidente Prudente, SP, Brazil \\ 5 Space Research Center, Polish Academy of Sciences, ul. Bartycka18a, 00-716 Warsaw, Poland \\ 6 Centre for Atmospheric Research, University of Nova Gorica, Vipavska 13, SI 5000 Nova Gorica, Slovenia \\ 7 Pildo Consulting, SL, Parc Tecnològic de Barcelona Nord Office A216-A220, Marie Curie 8-14, 08042 Barcelona, Spain \\ 8 Consultgel Consultoria em Geomatica Ltda, Rua Jose Tognoli, 238, Presidente Prudente, SP 19060-370, Brazil
}

Received 10 February 2011 / Accepted 24 October 2011

\begin{abstract}
Scintillations are rapid fluctuations in the phase and amplitude of transionospheric radio signals which are caused by small-scale plasma density irregularities in the ionosphere. In the case of the Global Navigation Satellite System (GNSS) receivers, scintillation can cause cycle slips, degrade the positioning accuracy and, when severe enough, can even lead to a complete loss of signal lock. Thus, the required levels of availability, accuracy, integrity and reliability for the GNSS applications may not be met during scintillation occurrence; this poses a major threat to a large number of modern-day GNSS-based applications. The whole of Latin America, Brazil in particular, is located in one of the regions most affected by scintillations. These effects will be exacerbated during solar maxima, the next predicted for 2013. This paper presents initial results from a research work aimed to tackle ionospheric scintillation effects for GNSS users in Latin America. This research is a part of the CIGALA (Concept for Ionospheric Scintillation Mitigation for Professional GNSS in Latin America) project, co-funded by the EC Seventh Framework Program and supervised by the GNSS Supervisory Authority (GSA), which aims to develop and test ionospheric scintillation countermeasures to be implemented in multi-frequency, multi-constellation GNSS receivers.
\end{abstract}

Key words. 2447: modelling and forecasting - 2400: ionosphere - 2415: equatorial ionosphere -2439 : ionospheric irregularities

\section{Introduction}

The Earth's ionosphere is the single largest contributor to the Global Navigation Satellite System (GNSS) error budget and the phenomenon of scintillation in particular poses degrading effects. Ionospheric scintillation is a rapid fluctuation in the amplitude and phase of radio signals from GNSS satellites as they pass through small-scale plasma density irregularities in the ionosphere (Wernik \& Liu 1974; Kintner et al. 2001 and the references therein). Scintillation may have a considerable impact on the performance of space geodesy, navigation and communication systems; they can lead to an increase in the probability of losing the GNSS signal lock as well as reduce the precision of the pseudorange and phase measurements. Although scintillations are unlikely to affect all GNSS satellites in view simultaneously, losing the signal lock on some of the satellites can affect the accuracy of the positioning solution. In general, scintillation effects are characterised by two indices, namely the amplitude scintillation index, $\mathrm{S}_{4}$, which is the standard deviation of the received power normalised by its mean value, and the phase scintillation index, SigmaPhi $\left(\sigma_{\phi}\right)$, which is the standard deviation of the detrended carrier phase. These indices are considered at every 1-min interval.
The occurrence of scintillation shows large day-to-day variations and is determined by the local time, season, latitude, longitude, solar and geomagnetic activity (Aarons 1982; Groves et al. 1997 and the references therein). The two geographic regions where scintillation occurs more predominantly are the equatorial bands extending from about $20^{\circ} \mathrm{N}$ to $20^{\circ} \mathrm{S}$ geomagnetic latitudes and the high latitude (auroral and polar cap) regions. However, in these two regions, the processes which produce scintillation are quite different, thereby leading to significant differences in the characteristics of the observed scintillation effects. The auroral and polar cap scintillations are mainly the result of geomagnetic storms which are associated with solar flares, coronal mass ejections and coronal holes. Equatorial scintillation, on the other hand, is mostly produced after the local sunset by the combined effects of the chemical recombination and the electrodynamic lifting of the $F$-region by the Pre-Reversal Enhancement (PRE) (Kelley 1989). As equatorial scintillation is strongly coupled to the Equatorial Ionisation Anomaly (EIA), it tends to become frequent and more severe during the solar maximum period, when the anomaly is at its greatest and the plasma density irregularities occur in a background of high ionisation density. 
The most severe scintillation is generally associated with the crests of the EIA, which are centred approximately $15^{\circ}$ in latitude on either side of the magnetic equator. Over the Brazilian longitudinal sector, the large magnetic declination angle coupled with the horizontal gradients in the electron density due to EIA produces important peculiarities in the irregularity distribution (Abdu et al. 1981; de Paula et al. 2007 and the references therein). Under the geomagnetically disturbed periods, the formation/inhibition of small-scale plasma density irregularities causing scintillation becomes highly complex, as recently presented by Muella et al. (2010), who discussed events that took place over Brazil during the Halloween storm of 29-30 October 2003.

With the increasing reliance of modern-life applications on GNSS, in particular on geodesy and navigation, the precise information on the occurrence characteristics of scintillation and an ability to predict scintillation have become increasingly important. Mitigation tools are necessary to minimise the effects of scintillation (including loss of lock and accuracy degradation) on the receiver's performance. The research presented in this paper is a part of the CIGALA (Concept for Ionospheric Scintillation Mitigation for Professional GNSS in Latin America) project, co-funded by the EC Seventh Framework Program and supervised by the GNSS Supervisory Authority (GSA). This project aims to understand the cause and implications of scintillation over low latitudes, model their effects and develop novel countermeasures to be implemented in multi-frequency, multi-constellation GNSS receivers. Moreover, since the occurrence of scintillation is strongly correlated to the solar activity, the forthcoming solar maximum, peak expected around 2013 , opens a great opportunity to collect and analyse scintillation data. This collected data can be used to develop accurate scintillation prediction and receiver tracking models that can be directly used to develop novel mitigation algorithms.

The overall methodology used in this research is described in Section 2. Section 3 presents some results on the validation of a newly designed scintillation monitor receiver and some initial results from the scintillation climatology study based on data collected in Brazil. A scintillation event recorded in Brazil by the newly designed scintillation monitor receiver is also presented in this section with some results on receiver tracking performance under scintillation. Section 4 contains the conclusion.

\section{Methodology}

Although the theoretical studies, intensive observations and refined modelling techniques have isolated the stabilising (Mendillo et al. 1992) and the destabilising forces (Basu et al. 1996) in the equatorial ionosphere, the forecasting of equatorial scintillation still remains a challenging task. Thus, research on the underlying causes of scintillation and the development of the state-of-the-art models capable of predicting signal propagation and tracking perturbations remains relevant. With that in mind this research work aims to take advantage of field measurements via the deployment of multi-frequency, multi-constellation GNSS receivers (Septentrio's PolaRxS receivers) at stations in Brazil. These receivers' specifications were laid out at the early stages of this research to provide the computation and storage of some parameters of interest for scintillation analysis and implementation of mitigation techniques. At the time of writing such receivers were only recently deployed. They will allow open sky data to be recorded, which can be analysed to support the development of the scintillation countermeasures to be implemented at receiver level. The following subsections give a brief description of the current status of this research.

\subsection{Scintillation and tracking modelling}

GNSS receivers need to robustly and continuously track the incoming satellite signals. Demodulation of the navigation data from the incoming signal requires an exact replica of the signal, which can be achieved by tracking the code and phase (or frequency) of the incoming signal through a Delay-Locked Loop and a Phase (or Frequency)-Locked Loop, PLL (or FLL), respectively. Ionospheric scintillation may cause the PLL to lock onto a wrong phase while tracking the signal, which can degrade the quality of the carrier phase measurements, or even lose lock completely, which causes cycle slips (Conker et al. 2003; Humphreys et al. 2005 and the references therein). The mean time between cycle slips depends on the intensity of scintillation such that under high scintillation levels, the PLL may never recover the phase lock. Under such conditions, the receiver cannot use the signal from that particular satellite, leading to poorer receiver-satellite geometry. This problem may intensify during solar maximum especially at the low latitudes, when the intensity of scintillation becomes stronger and the signals from a number of satellites may be affected simultaneously (Groves et al. 2000; Morrissey et al. 2004 and the references therein). Therefore, developing new ionospheric scintillation and receiver tracking models and/or an improvement of existing models are a necessity.

The receiver PLL performance is usually evaluated in terms of the phase error variance, which is observed to increase during ionospheric scintillation (Conker et al. 2003). Several efforts have been made to model the effects of ionospheric scintillation on the PLL performance. These can be summarised as: (i) simulation of time histories passed through software models of tracking loops (Cervera \& Knight 1998; Conker et al. 2003; Humphreys et al. 2005), (ii) evaluation of PLL performance using a Global Positioning System (GPS) signal simulator (Morrissey et al. 2004) and (iii) observation of receiver performance during actual events of scintillation in locations of interest (Knight \& Finn 1998; Groves et al. 2000). Although these approaches have provided some high-level description of the scintillation effects on the PLL performance, they also present some strong limitations if they were to be used to describe the picture completely (Humphreys et al. 2009).

The dependence of scintillation on local time, season, solar and magnetic activity has a stochastic character; therefore, the prediction of scintillation still remains a challenging task. Various models of scintillation have been developed to predict scintillation levels and therefore assist in mitigating this problem. The simulation of scintillation effects on the signals (to be input to the software PLL models) is accomplished by using either analytical models (Fremouw \& Rino 1973; Aarons 1985; Iyer et al. 2006), global climatological models like the WideBand MODel (WBMOD; Secan et al. 1995), Global Ionospheric Scintillation Model (Béniguel \& Buonomo 1999) or in situ data-based models (Basu et al. 1976; Wernik et al. 2007).

Based on a review of the available scintillation models, as presented in Aquino et al. (2010), it is suggested that the most 
V.V. Sreeja et al.: Tackling ionospheric scintillation threat

Table 1. List of the locations selected for the PolaRxS receivers' deployment. Co-locations with other instruments useful for the research are also reported.

\begin{tabular}{lcccc}
\hline \hline Name & Geo. latitude & Geo. longitude & GPS & Ionosonde \\
\hline Manaus & $3.1^{\circ} \mathrm{S}$ & $60.0^{\circ} \mathrm{W}$ & $\mathrm{Y}$ & $\mathrm{Y}$ \\
Porto Alegre & $30.0^{\circ} \mathrm{S}$ & $51.2^{\circ} \mathrm{W}$ & $\mathrm{Y}$ & $\mathrm{N}$ \\
Presidente Prudente & $22.1^{\circ} \mathrm{S}$ & $51.4^{\circ} \mathrm{W}$ & $45.1^{\circ} \mathrm{W}$ & $\mathrm{Y}$ \\
São José dos Campos & $22.2^{\circ} \mathrm{S}$ & $48.3^{\circ} \mathrm{W}$ & $\mathrm{Y}$ & $\mathrm{Y}$ \\
Palmas & $10.2^{\circ} \mathrm{S}$ & $41.8^{\circ} \mathrm{W}$ & $\mathrm{N}$ & $\mathrm{N}$ \\
Macaé & $22.3^{\circ} \mathrm{S}$ & & & \\
\hline
\end{tabular}

adequate models to predict the occurrence of scintillation within the context of this research, i.e. with the aim to aid GNSS satellite signal tracking under scintillation, are the WBMOD model (Secan et al. 1995) and the WAM (Wernik et al. 2007). Both these models rely on the physical principles driving the radio wave propagation through plasma density irregularities in the ionosphere according to specific heliogeophysical conditions. Although originally developed to model the high latitude ionospheric scintillation, the WAM will be modified to predict the occurrence of equatorial scintillation. This model will be adapted in the CIGALA to also output critical spectral parameters such as the spectral strength $(T)$ and slope $(p)$, which are needed for error analysis and for the implementation of improved receiver tracking models. Furthermore, the WAM can be updated and fine-tuned with the low latitude in situ satellite data (such as from the Communication/Navigation Outage Forecasting System).

Another approach to model ionospheric scintillation is by generating scintillation time histories that can be implemented in a GNSS hardware signal simulator, which can in turn be connected to a GNSS receiver to assess the effects on the receiver's tracking performance. One such model is the Cornell Scintillation Model (CSM), which is a statistical model that generates perturbations on the signal amplitude and phase (Humphreys et al. 2009), i.e. it is not a global scintillation model; it rather focuses on the severe equatorial scintillation effects on GPS signals.

\subsection{Instrumentation}

This research involves the collection of high sampling rate measurements of phase and amplitude of GNSS signals received over Latin America, covering as much as possible the equatorial region around the EIA crest locations. The monitoring stations are equipped with Septentrio's PolaRxS receivers, mentioned above. The list of the monitoring stations is given in Table 1 and a map showing their locations in Figure 1.

In the past, ionospheric monitoring was limited to the GPS L1C/A (Coarse/Acquisition code $-1575.42 \mathrm{MHz}$ ) and L2P (Precision code $-1227.60 \mathrm{MHz}$ ) signals. However, in recent years, there has been the advent of new civilian signals like the L2C (Coarse/Acquisition code $-1227.60 \mathrm{MHz}$ ) and L5 (1176.45 MHz) from the GPS satellites, L1 (1598.0625 1609.3125 MHz) and L2 (1242.9375 - $1251.6875 \mathrm{MHz})$ from GLONASS (Russian Global Navigation Satellite System) and L1 (1575.42 MHz), E5a (1176.45 MHz) and E5b $(1207.14 \mathrm{MHz})$ from the Galileo In-Orbit Validation Element (GIOVE) satellites. Therefore, the main novelty of this research is that scintillation effects on all these new signals will be monitored for the first time, using the PolaRxS receiver. Such monitoring will maximise the number of scintillation events that can be observed and will also increase the probability of actually recording the events. Furthermore, the signal structure used in the new GNSS signals is expected to lower the probability of loss of lock during scintillation, especially when the dataless component of these signals is tracked.

To support the research necessary to improve existing models or to develop new scintillation and receiver tracking models as proposed in Section 2.1, specifications were laid out and subsequently implemented on the PolaRxS receiver. This unit's main features, which are essential for this research, are summarised below.

The PolaRxS is a multi-frequency, multi-constellation GNSS receiver that incorporates a state-of-the-art triple frequency receiver engine that is capable of tracking simultaneously the GPS, GLONASS, Galileo and SBAS (SatelliteBased Augmentation System to GPS used for navigation and precision approaches) signals. It has an ultra-low noise oscillator frequency reference with a standard deviation of phase noise less than $0.03 \mathrm{rad}$. The receiver can generate and store raw high

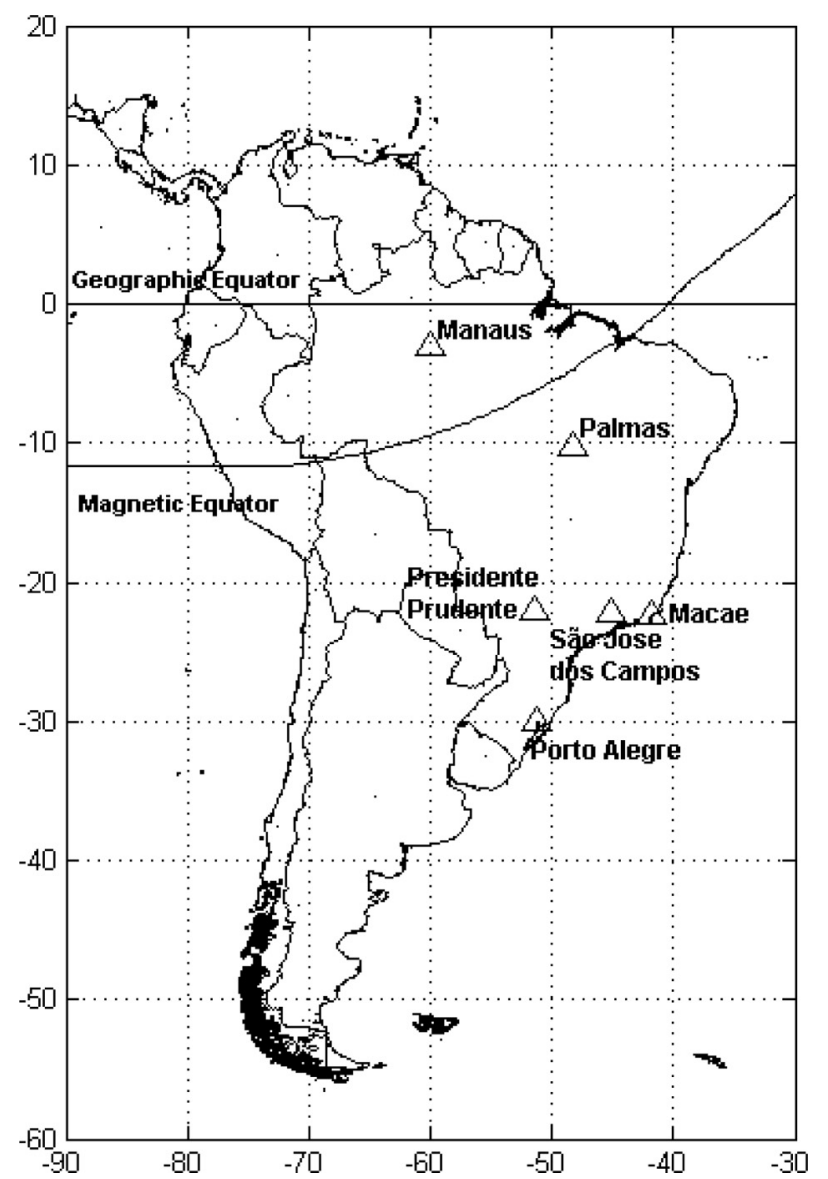

Fig. 1. Map showing the locations of the stations in Latin America. 
rate (correlated I and Q samples) data at $50 \mathrm{~Hz}$ in hourly files which can be (post- or real-time) processed to give $60 \mathrm{~s}$ scintillation indices, $\mathrm{S}_{4}$ and $\sigma_{\phi}$, along with other parameters like Total Electron Content, lock time and the scintillation spectral parameters, $p$ (spectral slope of the phase Power Spectral Density, PSD) and $T$ (spectral strength of the phase PSD at $1 \mathrm{~Hz}$ ), for all visible satellites and frequencies. The carrier frequencies that can be tracked are: GPS L1, L2, L5; GLONASS L1, L2; Galileo L1, E5a, E5b; SBAS L1 (1575.42 MHz). In addition, the new modulations, like GPS L2C and Galileo AltBOC (Alternative Binary Offset Carrier - $1191.795 \mathrm{MHz}$ ), can also be tracked on these carriers.

The high rate $(50 \mathrm{~Hz})$ as well as the 60 -s scintillation indices data will be used as input for the development of the new algorithms and models, firstly to reproduce and secondly to mitigate the scintillation effects.

\subsection{Mitigation strategy}

Based on the newly developed scintillation and receiver tracking models, a novel ionospheric scintillation countermeasure/ mitigation algorithm will be developed and implemented as part of the PolaRxS receiver firmware. As a first stage of this countermeasure/mitigation algorithm, the PLL performance under scintillation was investigated using the CSM. Simulated scintillation amplitude and phase time series were fed into a representative Matlab model of the tracking loop implemented in the PolaRxS receiver. It was found that, in the case of moderateto-strong scintillation, the probability of loss of lock could be significantly reduced by optimising the lock detector. The lock detector monitors the lock status of the loop and forces signal tracking to stop when the lock conditions are not met. The lock detector was too prudent in the sense that, in case of scintillation, loss of lock was declared while tracking could have continued without the loop diverging (a so-called false alarm). The lock detector has been optimised and receivers with the new firmware (improved lock detector threshold) will be field-tested in order to assess the improvement in robustness to real ionospheric scintillation. When they do not cause the receiver to lose lock, scintillation still introduces errors in GNSS positioning. This must be dealt with by suitable error modelling algorithms, using approaches such as, for instance, the one proposed in Aquino et al. (2009).

\section{Initial results}

Some of the preliminary results obtained from this research work are presented in the following subsections.

\subsection{Scintillation monitor validation}

In the past 10 years the GSV4000 series of receivers, developed by NovAtel and AJ Systems, has been widely used and well accepted by the scientific community to monitor GPS scintillation (Rama Rao et al. 2006; Aquino et al. 2009 and the references therein). The multi-frequency, multi-constellation Septentrio PolaRxS receiver was therefore validated against this unit.

In the validation process both receivers continually logged open sky data through a splitter connected to the same antenna (setup at the IESSG in Nottingham; geographic latitude $53^{\circ} \mathrm{N}$ ) for a period of $24 \mathrm{~h}$. Figure 2 shows a comparison in the 1-min scintillation indices, $\sigma_{\phi}$ (top panel) and $\mathrm{S}_{4}$ (bottom panel),
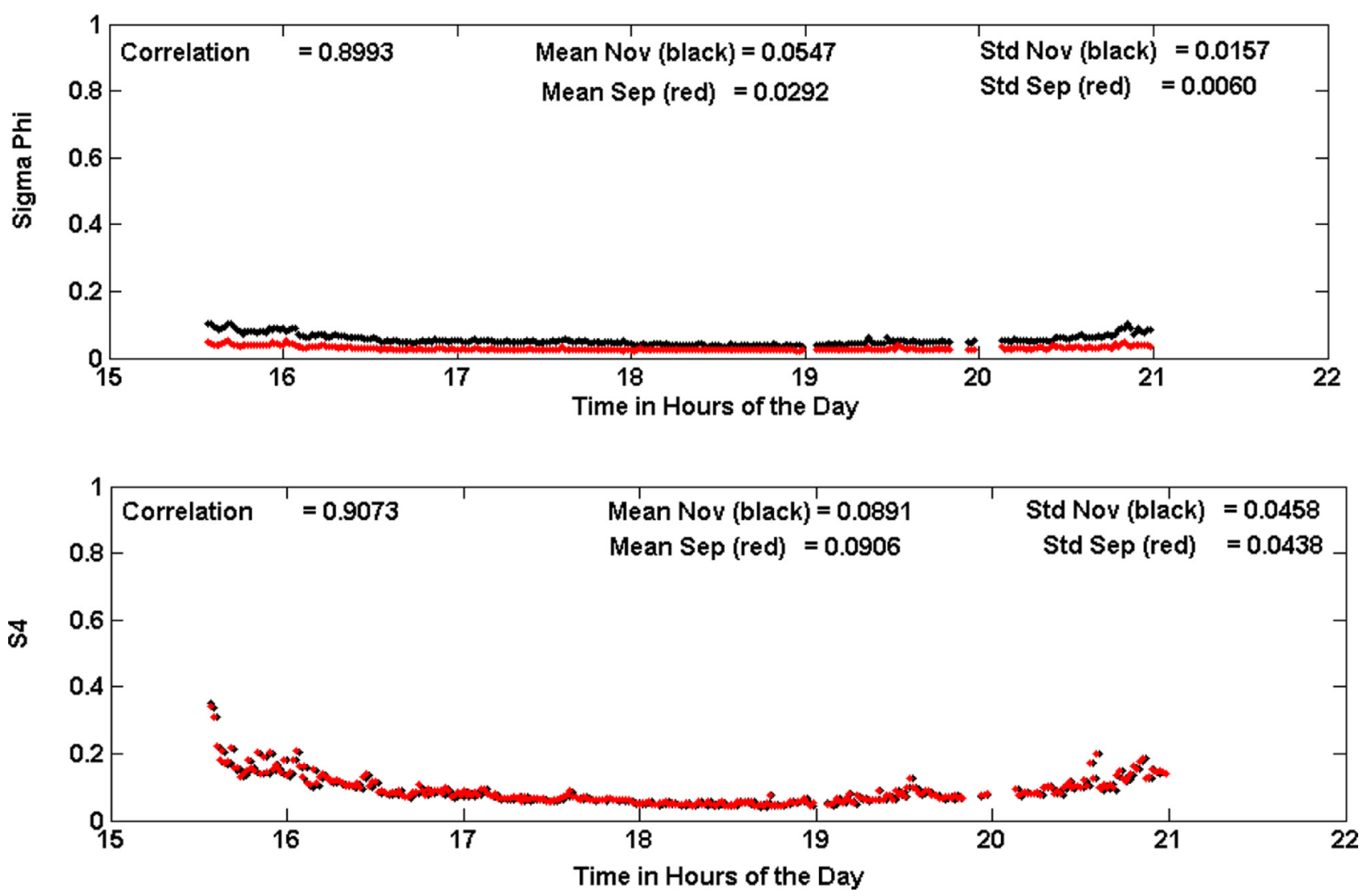

Fig. 2. Comparison of the scintillation indices, $\sigma_{\phi}$ and $\mathrm{S}_{4}$, recorded by the PolaRxS (red dots) and GSV4004 (black dots) receivers for the data collected from open sky for the GPS L1C/A signal of the satellite PRN19. 
estimated from open sky signals at Nottingham, for the GSV4004 (black marker) and PolaRxS (red marker) receivers, respectively, for the GPS L1C/A signal of satellite PRN19. This location was chosen, given the very quiet conditions of the midlatitude of Nottingham prevailing during the solar minimum period, which provides the right environment for a comparison of the sensitivity and noise floor of the two receivers. It is observed from the bottom panel of Figure 2 that $S_{4}$ indices recorded by both receivers are comparable, with a correlation coefficient of 0.9 . The mean value of the $\mathrm{S}_{4}$ index recorded by the PolaRxS receiver is slightly higher than that recorded by the GSV4004 receiver (mean value of PolaRxS $=0.0906$, mean value of GSV4004 $=0.0891$ ). This small offset in the $\mathrm{S}_{4}$ values is probably due to the change in signal-to-noise ratio introduced by the use of the splitter. From the top panel of Figure 2, it is observed that the $\sigma_{\phi}$ values recorded by the PolaRxS receiver are lower than those recorded by the GSV4004 receiver: the mean value of $\sigma_{\phi}$ for the PolaRxS receiver is 0.0292 and that for GSV4004 receiver is 0.0547 . This gives evidence that the PolaRxS receiver oscillator seems to provide a better noise floor level than its GSV4004 counterpart.

\subsection{Climatology studies based on data collected at the Brazilian station, Presidente Prudente (PP)}

An analysis with the Ground-Based Scintillation Climatology (GBSC) method was performed on the available data from PP $\left(22.1^{\circ} \mathrm{S}, 51.4^{\circ} \mathrm{W}\right.$, dip latitude $\left.=12.3^{\circ} \mathrm{S}\right)$ in Brazil. GBSC was originally developed for the high latitude receivers to investigate the physical process involved in the ionospheric scintillation, to contribute to the mitigation algorithms and as a first step towards forecasting the space-weather-related events with the GNSS receivers (Spogli et al. 2009, 2010; Alfonsi et al. 2011). The core of the GBSC is the maps of amplitude and, if available, phase scintillation occurrence. All the satellites in view at each epoch are considered to produce the maps. In the low latitude version of the GBSC, the maps can be defined in a bi-dimensional coordinate system expressed in terms of couples of two of the following: geographic coordinates, latitude and longitude, universal time, azimuth and elevation. The adopted binning is selected according to the available statistics and to a meaningful fragmentation of the map. The percentage occurrence $O$ of the given index is evaluated in each bin of the map as:

$$
O=N_{\text {thr }} / N_{\text {tot }}
$$

where $N_{\text {thr }}$ is the number of data points corresponding to the index above the characterising threshold and $N_{\text {tot }}$ is the total number of data points in the bin. Typical threshold values are 0.25 (in unit of the index) for moderate/strong scintillation scenarios and 0.1 for weak conditions. To remove the contribution of the bins with a scarce statistics, the data considered in each bin must be above a given number. To ensure this, we adopted a threshold on the statistical accuracy that is defined as (Taylor 1997):

$$
R_{\%}=100 \times \sigma\left(N_{\text {tot }}\right) / N_{\text {tot }}
$$

where $\sigma\left(N_{\text {tot }}\right)$ is the standard deviation of the number of data points in each bin and $N_{\text {tot }}$ is the total number of data points in the bin. Typical threshold values of $R_{\%}$ between $2.5 \%$ and $10 \%$ are a good compromise between the necessity to include meaningful bins in the map and to avoid overestimations of the occurrence due to scarce statistics.

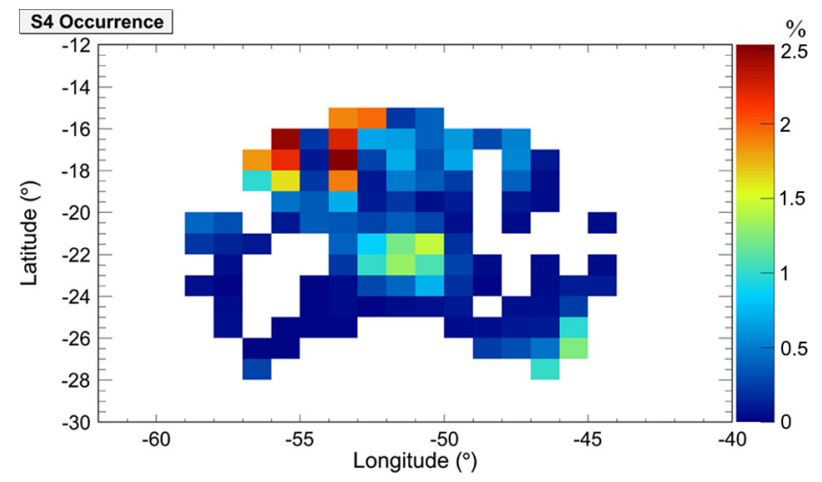

Fig. 3. Geographic latitude vs. Geographic longitude map of the percentage of occurrence of $\mathrm{S}_{4}>0.25$ for the investigated period in 2009.

This past data was collected by a SCINTMON (Cornell Scintillation Monitor) receiver (developed by Cornell University) (Beach \& Kintner 2001 and the references therein), managed by the Instituto Nacional de Pesquisas Espaciais (INPE, the Brazilian National Institute for Space Research), which has the ownership of the data. SCINTMON is a single frequency receiver that is not able to calculate the phase scintillation index, but only $\mathrm{S}_{4} . \mathrm{S}_{4}$ data analysed refer to the solar minimum year of 2009, from 1 January to 31 October, with a significant data gap between 29 June and 25 July.

Figure 3 shows a map (in geographic latitude and longitude) of the percentage of occurrence of $\mathrm{S}_{4}$ for the investigated period in a $1^{\circ} \times 1^{\circ}$ grid by selecting the threshold of $\mathrm{S}_{4}>0.25$, accuracy threshold is $5 \%$. Three regions of ionospheric scintillation in the field of view of the receiver in PP are revealed from this figure. The first one is located in the latitudinal range $20^{\circ}-15^{\circ} \mathrm{S}$ and in the longitudinal range $57^{\circ}-53^{\circ} \mathrm{W}$, corresponding to the southern crest of the EIA. The second and third regions are located $26-28^{\circ} \mathrm{S}$ and $45-47^{\circ} \mathrm{W}$ and about $22^{\circ} \mathrm{S}$ and $50-53^{\circ} \mathrm{W}$, respectively, with the former probably related to the signatures of the Southern Magnetic Anomaly (SAMA) (Abdu et al. 2005) and both being further investigated.

Further, a preliminary analysis on the early data acquired by the PolaRxS receiver in PP was done by applying the GBSC to the first two days of the data: 11-12 January 2011. The GBSC is also able to produce maps of the mean and standard deviation of the different quantities measured by the receiver, and, in this case, of the scintillation indices. These maps are typically constructed similarly to those for the occurrence using the same system of coordinates, bin size and accuracy. To produce the mean and standard deviation maps, the distribution of all the values of the investigated quantity is evaluated in each bin. The corresponding bins of the mean and standard deviation maps are then filled with the distribution mean value and standard deviation of the distribution, respectively.

The top panel of Figure 4 shows the azimuth versus elevation map of the $\mathrm{S}_{4}$ mean values (bin size: $10^{\circ} \times 5^{\circ}$ ) and the bottom panel shows the corresponding $\mathrm{S}_{4}$ standard deviation values in the same coordinate system and map segmentation, but without applying the elevation cut. The $\mathrm{S}_{4}$ index has been evaluated on the L1 measurement from both GPS and GLONASS constellations, using for the first time both the constellation measurements for climatological purpose. Both the mean and standard deviation values enhance at low elevation angles $\left(<20^{\circ}\right)$, probably because of non-ionospheric effects on tracking errors (like multipath) and confirming the necessity to 

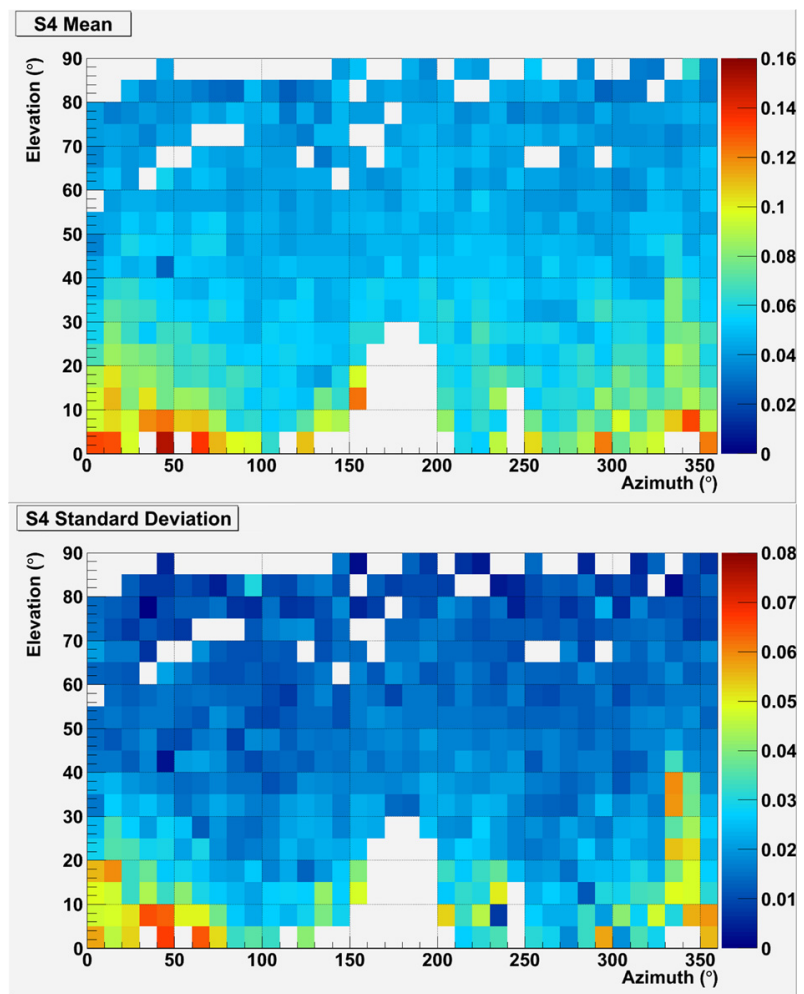

Fig. 4. Maps of the amplitude scintillation index $\mathrm{S}_{4}$ measured on L1 frequency in azimuth vs. elevation. Top plot shows the mean values, bottom plot shows the root mean square.

remove contribution from such low elevation angle measurements. Just above the $20^{\circ}$ elevation, the mean value increases in the azimuthal range of about $\left(330^{\circ}, 360^{\circ}\right)$ and $\left(0^{\circ}, 30^{\circ}\right)$, i.e. in the N-NW direction. In correspondence, also the standard deviation increases, indicating a region where larger values of $\mathrm{S}_{4}$ are more probable. This region corresponds to the southern crest of the EIA, confirming what was already found with the historical data analysis of the SCINTMON receiver. A longer time interval and availability of the data from the entire network of receivers, shown in Figure 1, are expected to maximise the number of scintillation events recorded and a more precise comparison with what was found with the SCINTMON historical data will be allowed. Moreover, the possibility of acquiring the signals from GPS (civil signals on L1, L2 and L5), GLONASS (civil signals on L1 and L2) and GALILEO/GIOVE (civil signals in the L1, E5a and E5b bands) increases the redundancy of the problem. All these features will allow an experimental identification of the different areas of the equatorial ionosphere more affected by the scintillation phenomena (and in particular the contribution of the SAMA) and their dependence on the local time and season.

\subsection{Scintillation event recorded by the PolaRxS receiver at PP}

The PolaRxS receiver deployed in PP recorded a scintillation event on 26 March 2011, a geomagnetically quiet day ( $K p$ value of 1). Figure 5 shows the variation in the scintillation indices $\left(\mathrm{S}_{4}-\right.$ bottom panel and $\sigma_{\phi}-$ top panel) as a function of local time and Ionospheric Pierce Point (IPP) latitude, during the post-sunset hours and for the GPS L1C/A signal. The IPP latitude for the different satellite-to-receiver links at every 1 min has been calculated assuming a single shell ionospheric model centred at an altitude of $350 \mathrm{~km}$. Also, a satellite

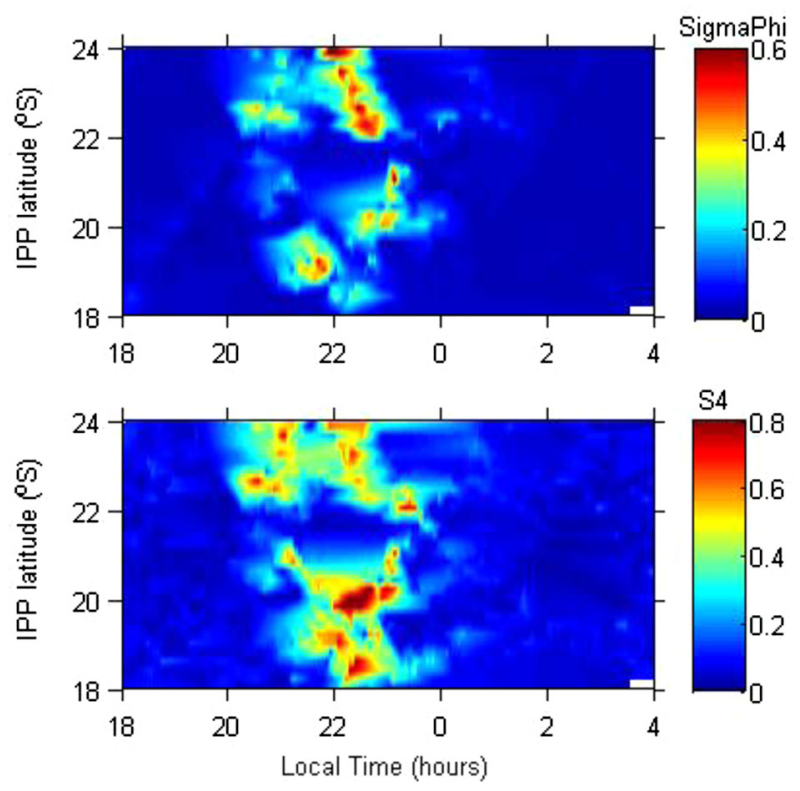

Fig. 5. Scintillation indices $\left(\mathrm{S}_{4}-\right.$ bottom panel and $\sigma_{\phi}-$ top panel) maps as a function of the local time and IPP latitude for 26 March 2011 for the GPS L1C/A signal.

elevation angle cut-off of $30^{\circ}$ is applied in order to reduce the impact of non-scintillation-related tracking errors (e.g. induced by multipath). Since scintillation events over the equatorial and low latitudes are essentially post-sunset phenomena, the local time in Figure 5 varies continuously from 18:00 LT to next morning 04:00 LT. It can be observed from the enhancement in $\mathrm{S}_{4}$ and $\sigma_{\phi}$ that, the evolution of the irregularities on this day occurred between 20:00 and 24:00 LT. Further, $\mathrm{S}_{4}$ and $\sigma_{\phi}$ values are considerably higher (around 0.8 and 0.6 , respectively) and more widespread in latitude, implying that the irregularities have a greater latitudinal extent (extending between 18 and $24^{\circ} \mathrm{S}$ ).

\subsection{Receiver tracking performance}

Once validated for use in scintillation monitoring, the PolaRxS receiver tracking performance was evaluated. This was done by calculating the variance of the error at the output of the PLL using the formula given in Conker et al. (2003). The Conker et al. (2003) formula for the GPS L1 carrier PLL accounts for the effects of scintillation on the input phase and computes the tracking error variance at the output of the PLL $\left(\sigma_{\phi}^{2}\right)$ as:

$$
\sigma_{\phi}^{2}=\sigma_{\phi_{s}}^{2}+\sigma_{\phi_{T}}^{2}+\sigma_{\phi_{\text {osc }}}^{2}
$$

where $\sigma_{\phi_{s}}^{2}, \sigma_{\phi_{T}}^{2}$ and $\sigma_{\phi_{\text {osc }}}^{2}$ are the error variance components relating to the phase scintillation, the thermal noise (amplitude scintillation) and the oscillator noise (assumed as $0.01 \mathrm{rad}^{2}$ in the receiver), respectively.

In equation (3), amplitude scintillation is modelled as an increase in the thermal noise, related to the decrease in the received signal power as (Conker et al. 2003):

$$
\sigma_{\phi_{T}}^{2}=\frac{B_{n}\left[1+\frac{1}{2_{\eta}\left(c / \eta_{0}\right)_{\mathrm{L} 1-C / A}\left(1-2 \mathrm{~S}_{4}^{2}(\mathrm{~L} 1)\right)}\right]}{\left(c / \eta_{0}\right)_{\mathrm{L} 1-C / A}\left(1-\mathrm{S}_{4}^{2}(\mathrm{~L} 1)\right)}
$$

where $B_{n}$ is the L1 third-order PLL one-sided bandwidth equal to $15 \mathrm{~Hz},\left(c / \eta_{0}\right)_{\mathrm{L} 1-C / A}$ is the fractional form of signal- 
V.V. Sreeja et al.: Tackling ionospheric scintillation threat
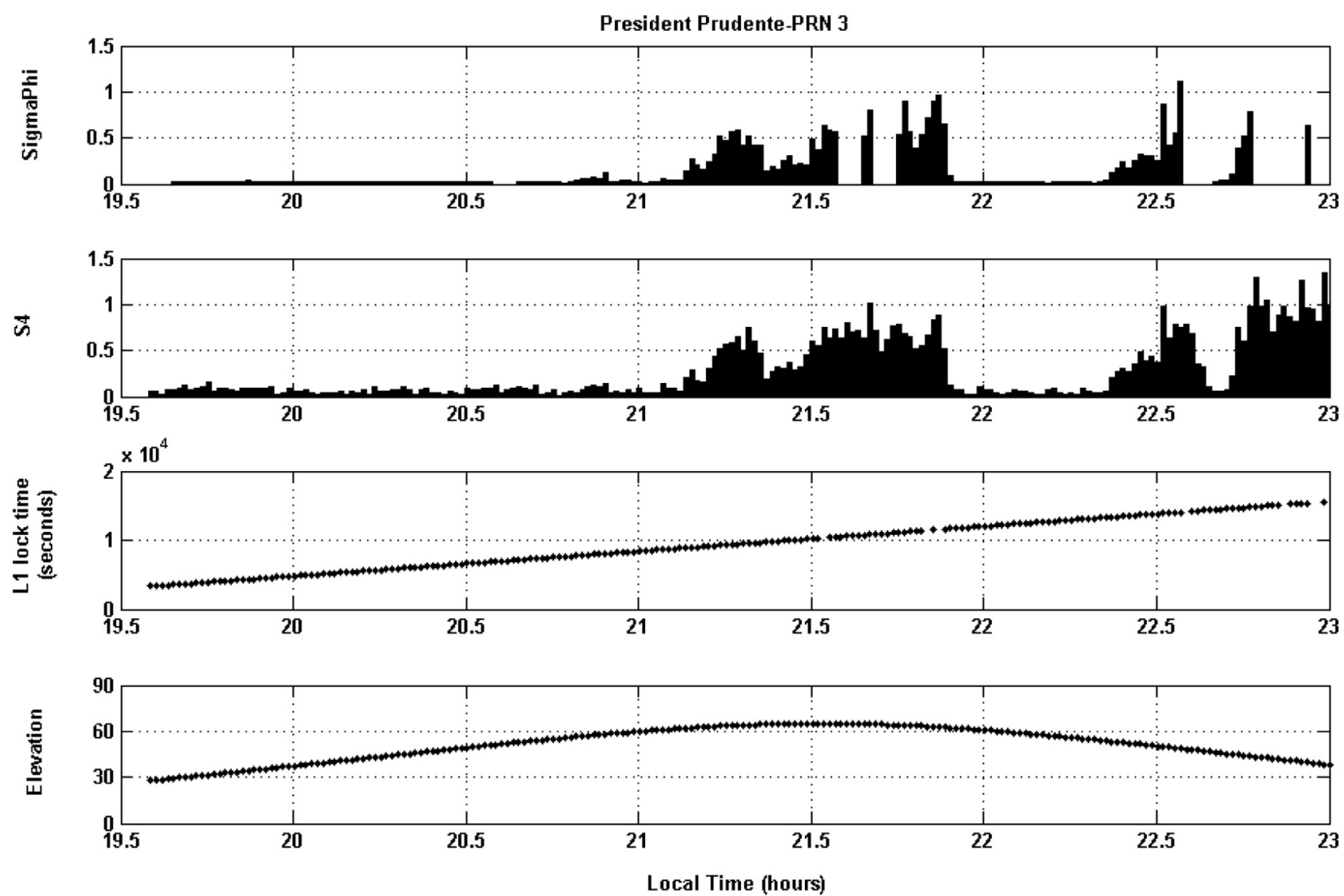

Fig. 6a. Variation in the scintillation indices $\sigma_{\phi}$, and $\mathrm{S}_{4}$ for GPS PRN3 observed by PolaRxS receiver on 11 March 2011.

to-noise density ratio, equal to $10^{0.1 \mathrm{C} / \mathrm{N} 0}, \eta$ is the predetection integration time, equal to $0.01 \mathrm{~s}$ and $\mathrm{S}_{4}(\mathrm{~L} 1)$ is the amplitude scintillation index measured at L1 frequency. The model is valid only for $\mathrm{S}_{4}(\mathrm{~L} 1)<0.707$; loss of lock is assumed for greater values of $\mathrm{S}_{4}$.

The phase scintillation component is modelled as (Conker et al. 2003):

$$
\sigma_{\phi_{s}}^{2}=\frac{\pi T}{k f_{n}^{p^{-1}} \sin \left(\frac{[2 k+1-p] \pi}{2 k}\right)}
$$

where $T$ is the spectral strength of the phase PSD at $1 \mathrm{~Hz}, p$ is the spectral slope of the phase PSD, $k$ is the order of the PLL (equal to 3 ) and $f_{n}$ is the loop natural frequency (equal to $1.91 \mathrm{~Hz})$.

Figure $6 \mathrm{a}$ shows the time variations of the 1-min scintillation indices $\left(\mathrm{S}_{4}\right.$ and $\sigma_{\phi}-$ top two panels), lock time on the GPS L1 carrier phase (third panel from top) and the elevation angle (bottom panel) as recorded by the PolaRxS receiver in PP for the satellite PRN3 on 11 March 2011. It can be observed from this figure that the value of the scintillation indices $\left(\mathrm{S}_{4}\right.$ and $\sigma_{\phi}$ ) is considerably higher (close to 1 ) along this satellite-toreceiver link.

The tracking error variance at the receiver PLL output for satellite PRN3 is calculated using equation (3). However, as explained earlier, this formula is limited to weak-to-moderate levels of scintillation $\left(\mathrm{S}_{4}<0.707\right)$ and hence cannot be applied for all levels of scintillation, even if the receiver does not lose lock and provide the required parameters needed to proceed with the calculations. The top panel in Figure $6 \mathrm{~b}$ shows the variations in the tracking jitter variance estimated for GPS
L1C/A of satellite PRN3. The middle panel shows the variation of the jitter variance with $\mathrm{S}_{4}$ and the bottom panel shows the variation of the jitter variance with $\sigma_{\phi}$ The gaps in the figures correspond to times when the jitter calculation by the formulae by Conker et al. (2003) is not valid due to $S_{4}>0.7$. It is observed from Figure $6 \mathrm{~b}$ that the tracking jitter variance increases with an increase in $\mathrm{S}_{4}$ as well as $\sigma_{\phi}$. The exact functional dependence of the tracking jitter variance on the scintillation indices is under investigation.

\section{Conclusion}

The overall methodology adopted in a research work aimed to tackle ionospheric scintillation effects for GNSS users in Latin America is described in this paper. The fundamental threat to the accuracy, integrity and availability of GNSS in general, and Galileo-related applications in particular, will be addressed through this research. This is crucial in Brazil, where scintillations significantly impact GNSS-based applications.

The initial validation tests of the Septentrio PolaRxS receiver reveal good agreement in the scintillation indices recorded by the PolaRxS and GSV4004 receivers indicating that the performance of the PolaRxS receiver is comparable to that of the GSV4004 receiver and can be effectively deployed for the scintillation studies. The preliminary results obtained with the GBSC method applied on the data acquired by the SCINTMON and the PolaRxS receivers at PP in Brazil reveal an enhancement in the scintillation occurrence that is observed to be co-located with the southern EIA crest, as is expected for the equatorial ionosphere. This indicates that the data 

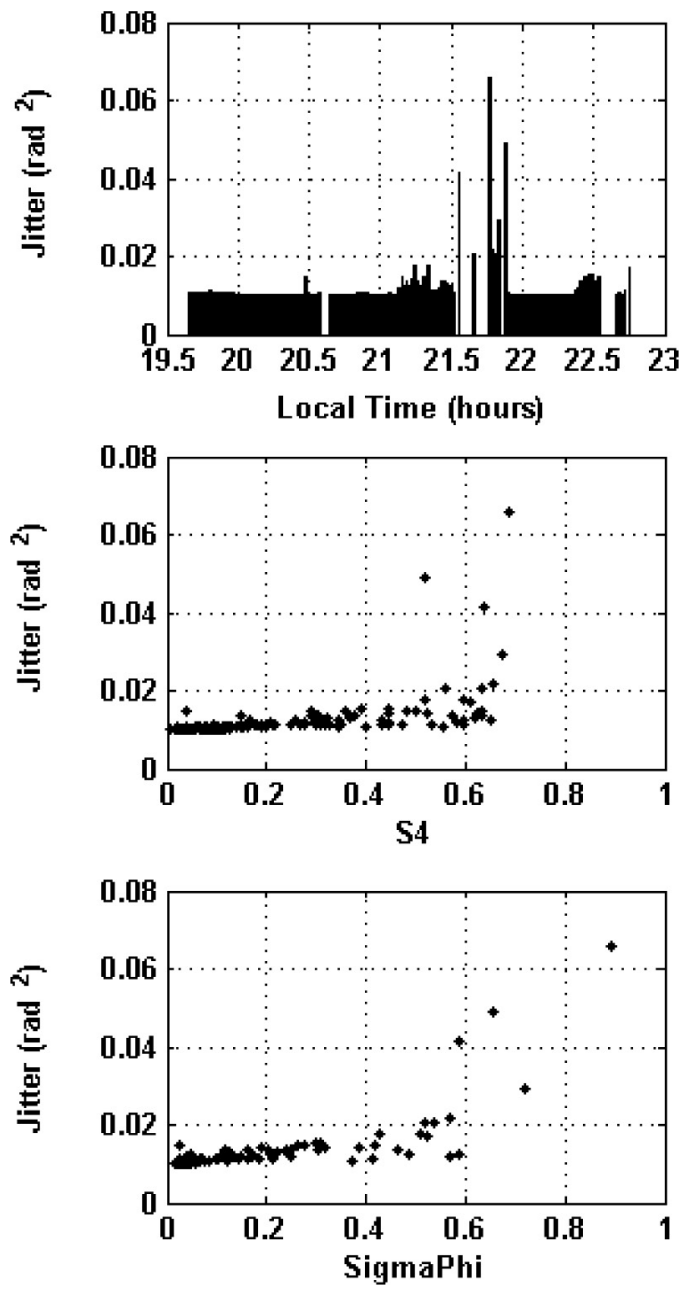

Fig. 6b. Time variation of the tracking jitter variance estimated using the formula of Conker et al. (2003) for GPS L1C/A from satellite PRN3 on 11 March 2011. Middle panel: Variation of the tracking jitter variance as a function of the amplitude scintillation index, $\mathrm{S}_{4}$. Bottom panel: Variation of the tracking jitter variance as a function of the phase scintillation index, $\sigma_{\phi}$.

collected by the deployment of the receivers at locations close to the EIA crest in Latin America can contribute to a better understanding of the scintillation occurrence characteristics and statistics, and can be used in developing a prediction/mitigation tool for the scintillation events at these latitudes. The PolaRxS receiver tracking performance evaluated by calculating the tracking jitter variance was found to depend on the scintillation level, more explicitly through the scintillation indices, $\mathrm{S}_{4}$ and $\sigma_{\Phi}$. Performance analyses of the receivers for moderate and strong levels of scintillation are investigated so that the tracking performance of the receivers can be optimised against the degrading effects of the low latitude scintillation effects.

Acknowledgements. The CIGALA project is funded under the EC Seventh Framework Program and is carried out in the context of the Galileo FP7 R\&D program supervised by the GSA. The authors thank Prof. Eurico De Paula of the Instituto Nacional de Pesquisas Espaciais (INPE) for providing a representative dataset collected through a SCINTMON unit. Biagio Forte's research activity at the University of Nottingham is funded by a EC FP7 Marie Curie Intra-European Fellowship.

\section{References}

Aarons, J., Global morphology of ionospheric scintillations, Proc. IEEE, 70, 360-378, 1982.

Aarons, J., Construction of a model of equatorial scintillation intensity, Radio Sci., 463 (20), 397-402, 1985.

Abdu, M.A., I.S. Batista, A.J. Carrasco, and C.G.M. Brum, South Atlantic Magnetic anomaly ionization: A review and a new focus on electrodynamics effects in the equatorial iono-sphere, J. Atm. Solar-Terr. Phys., 67, 1643-1657, DOI: 10.1016/j.jastp.2005.01.014, 2005.

Abdu, M.A., J.A. Bittencourt, and I.S. Batista, Magnetic declination of the 466 equatorial $\mathrm{F}$ region dynamo electric field development and spread F, J. Geophys. Res., 86 (467), 11443-11446, 1981.

Alfonsi, L., L. Spogli, G. De Franceschi, V. Romano, M. Aquino, A. Dodson, and C.N. Mitchell, Bipolar climatology of GPS ionospheric scintillation at solar minimum, Radio Sci., 46, RS0D05, DOI: 10.1029/2010RS004571, 2011.

Aquino, M., Z. Elmas, B. Forte, G. De Franceschi, L. Alfonsi, A. Wernik, and A. Canton, State of the art review, CIGALA D2.1WP200/V1.0 Final Version, http:/www.galileoic.org/la/?q=node/ 322,2010

Aquino, M., J.F.G. Monico, A. Dodson, H. Marques, G. De Franceschi, L. Alfonsi, V. Romano, and M. Andreotti, Improving the GNSS positioning stochastic model in the presence of ionospheric scintillation, J. Geodesy., 83 (10), 953-966, DOI: 10.1007/s00190-009-0313-6, 2009.

Basu, Su., Sa. Basu, and B.K. Khan, Model of equatorial scintillation from in-situ measurements, Radio Sci., 11, 821-832, 1976.

Basu, S., E. Kudeki, Su. Basu, C.E. Valladares, E.J. Weber, et al., Scintillations, plasma drifts and neutral winds in the equatorial ionosphere after sunset, J. Geophys. Res., 101, 795-26809, 1996.

Beach, T.L., and P.M. Kintner, Development and use of a GPS ionospheric scintillation monitor, IEEE Trans. Geosci. Remote Sens. (USA), 39, 918-928, 2001.

Béniguel, Y., and S. Buonomo, A multiple phase screen propagation model to estimate fluctuations of transmitted signals, Phys. Chem. Earth (C), 24, 333-338, 1999.

Cervera, M.A., and M. Knight, Time series modeling of intensity and phase 512 scintillation at GPS frequencies, Acta Geod. Geophys. Hung., 33 (1), 25-40, 513-514, 1998.

Conker, R.S., M.B. El Arini, C.J. Hegarty, and T. Hsiao, Modeling the effects of ionospheric scintillation on GPS/SBAS availability, Radio Sci., 38 (1), DOI: 10.1029/2000RS002604, 2003.

de Paula, E.R., E.A. Kherani, M.A. Abdu, I.S. Batista, J.H.A. Sobral, et al., Characteristics of the ionospheric irregularities over Brazilian longitudinal sector, IJRSP, 36, 268-277, 2007.

Fremouw, E.J., and C.L. Rino, An empirical model for average F-layer scintillation at VHF/UGF, Radio Sci., 8, 213-222, 1973.

Groves, K.M., S. Basu, E.J. Weber, M. Smitham, H. Kuenzler, et al, Equatorial scintillation and systems support, Radio Sci., 32, 2047-2064, 1997.

Groves, K.M., S. Basu, J.M. Quinn, T.R. Pedersen, K. Falinski, A. Brown, R. Silva, and P. Ning, A comparison of GPS performance in a scintillating environment at Ascension Island, Proceedings of ION GPS 2000, Institute of Navigation, Salt Lake City, UT, 2000.

Humphreys, T.E., M.L. Psiaki, B.M. Ledvina, and P.M. Kintner, GPS carrier tracking loop performance in the presence of ISs, Proc. ION GNSS 2005, Institute of Navigation, Long Beach, CA, 2005.

Humphreys, T.E., M.L. Psiaki, J.C. Hinks, B. O'Hanlon, and P.M. Kintner Jr., Simulating ionosphere-induced scintillation for testing GPS receiver phase tracking loops, IEEE J. Select. Topics Signal Process., 3, 707-715, 2009.

Iyer, K.N., J.R. Souza, B.M. Pathan, M.A. Abdu, M.N. Jivani, and H.P. Joshi, A model of equatorial and low latitude VHF scintillation in India, Indian J. Radio \& Space Phys., 35, 98104, 2006.

Kelley, M.C., The Earth's Ionosphere: Plasma Physics and Electrodynamics, Academic Press, San Diego, CA, 1989. 
V.V. Sreeja et al.: Tackling ionospheric scintillation threat

Kintner, P.M., H. Kil, T.L. Beach, and E.R. de Paula, Fading Timescales Associated with GPS Signals and Potential Consequences, Radio Sci., 36 (4), 731-743, 2001.

Knight, M., and A. Finn, The effects of ionospheric scintillation on GPS, Proc. ION GPS 1998, Nashvill, TN: ION, 1998.

Mendillo, M., J. Baumgardner, Pi. Xiaoquing, P.J. Sultan, and R.T. Tsunoda, Onset conditions for equatorial spread F, J. Geophys. Res., 97 (13), 865-876, 1992.

Morrissey, T.N., K.W. Shallberg, A.J. Van Dierendonck, and M.J. Nicholson, GPS receiver performance characterization under realistic ionospheric phase scintillation environments, Radio Sci., 39, 1-18, 2004.

Muella, M.T.A.H., E.A. Kherani, E.R. de Paula, A.P. Cerruti, P.M. Kintner, I.J. Kantor, C.N. Mitchell, I.S. Batista, and M.A. Abdu, Scintillation-producing Fresnel-scale irregularities associated with the regions of steepest TEC gradients adjacent to the equatorial ionization anomaly, J. Geophys. Res., 115, A03301, DOI: 10.1029/2009JA014788, 2010.

Rama Rao, P.V.S., S. Gopikrishna, K. Niranjan, and D.S.V.V.D. Prasad, Study of spatial and temporal characteristics of L-band scintillations over the Indian low latitude region and their possible effects on GPS navigation, Ann. Geophys., 24, 1567-1580, 2006.
Secan, J.A., R.M. Bussey, E.J. Fremouw, and Sa. Basu, An improved model of equatorial scintillation, Radio Sci., 30, 607-617, 1995.

Spogli, L., L. Alfonsi, G. De Franceschi, V. Romano, M.H.O. Aquino, and A. Dodson, Climatology of GPS ionospheric scintillations over high and mid-latitude European regions, Ann. Geophys., 27, 3429-3437, 2009.

Spogli, L., L. Alfonsi, G. De Franceschi, V. Romano, M.H.O. Aquino, and A. Dodson, Climatology of GNSS ionospheric scintillations at high and mid latitudes under different solar activity conditions, Il Nuovo Cimento B, DOI: 10.1393/ncb/i2010-10857-7, 2010.

Taylor, J.R., An Introduction to Error Analysis: The Study of Uncertainties in Physical Measurement, 2nd edition, Univ. Sci., Sausalito, CA, 1997.

Wernik, A.W., L. Alfonsi, and M. Materassi, Scintillation modeling using in situ data, Radio Sci., 42, RS1002, DOI: $10.1029 / 2006 \mathrm{RS} 003512,2007$.

Wernik, A.W., and C.H. Liu, Ionospheric irregularities causing scintillations of $\mathrm{GHz}$ frequency radio signals, J. Atm. Terr. Phys., 36, 871-879, 1974. 